\section{Virtuelle Darmspiegelung ist Screening-tauglich}

\begin{abstract}
Das Angebot einer virtuellen Darmspiegelung (CT-Kolonografie) erhöht die Teilnahmeraten am Darmkrebsscreening signifikant. Die reale Koloskopie
\end{abstract} entdeckt zwar mehr fortgeschrittene Neoplasien, doch auf die Screeningpopulation bezogen ist die diagnostische Ernte bei beiden Methoden gleich.

n einer Studie wurde der diagnostische

Ertrag von Koloskopie und CT-Kolonografie (ohne Darmreinigung, mit iodiertem Kontrastmittel) miteinander verglichen. Er errechnete sich jeweils als die Zahl entdeckter fortgeschrittener Geschwülste pro untersuchte Personen. Als fortgeschritten

galten Adenome $\geq 10$ mm oder mit einem 25\%igen villösen Anteil sowie Karzinome.

Zur Koloskopie geladen wurden 5.924 Niederländer, davon nahmen 1.276 (22\%) teil. 2.920 Personen wurde eine CT-Kolonografie angeboten, und 982 (34\%) nutzten das - eine Steigerung der Scree-

\begin{tabular}{|l|c|c|}
\hline $\begin{array}{l}\text { Komplikationen von } \\
\text { Probanden } 30 \text { Tage nach Koloskopie bzw. CT-Kolonografie }\end{array}$ & \\
\hline Unerwünschte Wirkungen & $\begin{array}{c}\text { Kolonoskopie } \\
(\mathbf{n = 1 . 2 7 6 )}\end{array}$ & $\begin{array}{c}\text { CT-Kolonografie } \\
\text { (n= 982) }\end{array}$ \\
\hline Post-Polypektomie-Blutungen & 2 & 3 \\
\hline Vorhofflimmern & 1 & 0 \\
\hline Kollaps & 1 & 1 \\
\hline Pneumonie & 1 & 0 \\
\hline Spinaler epiduraler Abszess & 1 & 0 \\
\hline Akutes Koronarsyndrom & 0 & 1 \\
\hline Akutes rheumatisches Fieber & 0 & 1 \\
\hline Apoplexie & 0 & 1 \\
\hline Myokardinfarkt & 0 & 1 \\
\hline Harnwegsinfektion & 1 & 0 \\
\hline Aufnahme des Desinfektionsmittels (30\%-Alkohol) statt des & 0 & 1 \\
\hline 50 ml iodierten Kontrastmittels & & \\
\hline
\end{tabular}

\section{Einmal Sigmoidoskopie}

Britische Ergebnisse, wonach Darmkrebsinzidenz und -mortalität durch eine einmalige Sigmoidoskopie gesenkt werden, wurden jetzt durch eine italienische Studie bestätigt.

Siling echs gastroenterologische Zentren luden 236.568 Männern und Frauen im Alter zwischen 55 und 64 Jahren zur Teilnahme ein. 56.532 Interessierten wurde 1995-1999 entweder eine flexible Sigmoidoskopie angeboten $(n=17.148)$, oder der Kontakt wurde nicht weitergeführt (n $=17.144$ ). Letztlich unterzogen sich 9.911 Personen dem Screening. Nach median 10,5 (für Inzidenzen) bzw. 11,4 Jahren (für ningquote um $56 \%(\mathrm{p}<0,0001) .9 \%$ der Koloskopie-Teilnehmer hatten eine fortgeschrittene Neoplasie, < $1 \%$ ein Karzinom. $8 \%$ der virtuell Gespiegelten wurde wegen kleinerer Läsionen eine FolgeKolonografie angeboten, $9 \%$ wurden wegen verdächtiger Befunde koloskopiert. $6 \%$ wiesen fortgeschrittene Tumoren auf, $<1 \%$ ein Karzinom.

Bezogen auf 100 Teilnehmer lag der Ertrag der Koloskopie bei 8,7, jener der Kolonografie bei 6,1 - die Koloskopie ist damit die genauere Methode. Bezogen auf die zum Screening Eingeladenen war die Koloskopie nicht erfolgreicher; ihr Ertrag lag bei 1,9/100 vs. 2,1/100 für die Kolonografie. Für Neoplasien von $\geq 10 \mathrm{~mm}$ lag der Ertrag/100 Geladenen bei 1,5 (Koloskopie) bzw. 2,0 (Kolonografie). Die Unterschiede waren nicht signifikant.

Fazit: „Beide Methoden eignen sich fürs populationsbezogene Screening“, konstatierten die Autoren. Aber auch die Belastung für die gescreenten Personen und die Kosteneffektivität müssten mit einfließen. Beispielsweise sind für eine Koloskopie rund 90 Euro und für die CT-Kolonografie je nach Methode etwa 400 Euro zu bezahlen.

Robert Bublak

Stoop EM et al. Participation and yield of colonoscopy versus non-cathartic CT colonography in population-based screening for colorectal cancer: a randomised controlled trial. Lancet Oncol. 2011;13(1):55-64.

denz reduzierte sich um $31 \%$ (RR 0,69; 95\%-KI 0,56-0,86), die Mortalität um $38 \%$ (RR 0,62; 95\%-KI 0,40-0,96).

Fazit: Eine einzige flexible Sigmoidoskopie zwischen 55 und 64 Jahren kann Inzidenz und Mortalität des CRC reduzieren. Ein Kommentator hält es zurzeit für wichtig, die möglichen Strategien mit dem Patienten zu diskutieren. Friederike Klein

Segnan N et al. Once-only sigmoidoscopy in colorectal cancer screening: follow-up findings of the Italian randomized controlled trial--SCORE. J NatI Cancer Inst. 2011; 103(17):1310-22. - Editorial: Church TE. Screening for colorectal cancer - which strategy is the best? Ibidem 1282-3. 